\title{
Comparison of the effects of adenosine, isoproterenol and their combinations on pulmonary transit time in rats using contrast echocardiography
}

\author{
Feng Su${ }^{1}$, Yun-Yan Shi ${ }^{1}$, Bo Wang ${ }^{1}$, Xiao-Zhi Zheng ${ }^{1,2}$ \\ ${ }^{1}$ Department of Ultrasound, Yancheng Clinical College of Xuzhou Medical University, Yancheng, Jiangsu Province, \\ ${ }^{2}$ Department of Ultrasound, Yangpu Hospital, Tongji University School of Medicine, Shanghai, China
}

\begin{abstract}
Aims: To compare the effects of adenosine (Ade), isoproterenol (Iso) and their combinations on pulmonary transit time (PTT) in rats using contrast echocardiography. Material and methods: Thirty-two adult Sprague Dawley (SD) rats were divided into four groups $(\mathrm{n}=8)$ according the medicines of tail-intravenous injection: Group 1, control; Group 2, Ade; Group 3, Iso; Group 4, Ade+Iso. They all underwent conventional echocardiography and contrast echocardiography with measurements of PTT. Results: With Ade injection, Onset $_{\mathrm{RV}}-\mathrm{Onset}_{\mathrm{LV}}$ PTT (PTT1), Peak $\mathrm{RV}-\mathrm{Peak}_{\mathrm{LV}}$ PTT (PTT2) and Onset ${ }_{\mathrm{RV}}-\mathrm{Peak}_{\mathrm{LV}}$ PPT (PTT3) decreased and PTT3 had the largest decreased percentage, with the highest performance in differentiating the Ade group from the control group [the area under receiver operating characteristic curve (AUC), sensitivity and Youden's index was maximal]. With Iso injection, PTT1, PTT2 and PTT1 all increased and PTT1 had the largest increased percentage, with the highest performance in differentiating the Iso group from the control group (AUC, sensitivity and Youden's index was maximal). With a combination injection of Ade and Iso, the PTT values were similar to the control group and no PTT could differentiate the Ade+Iso group from the control group. Conclusions: Ade or/and Iso exerted distinct effects on PTT. These findings remind us that it is a necessary to consider the effects of medicine (especially cardiopulmonary vasoactive drugs) on the PTT values. At the same time, it provides the basis for the clinical transformation of consecutive Iso/Ade treatment from the perspective of pulmonary circulation.
\end{abstract}

Keywords: pulmonary transit time; adenosine; isoproterenol; contrast echocardiograghy; rat

\section{Introduction}

Pulmonary transit time (PTT), defined as the time required for a volume of contrast to travel from the right ventricle $(\mathrm{RV})$ to the left atrium (LA) or the left ventricle (LV), has been reported to be a metric for integrative

Received 15.02.2021 Accepted 16.06.2021

Med Ultrason

2021:0 Online first, 1-7

Corresponding author: Xiao-Zhi Zheng, M.D, Ph.D

Department of Ultrasound, Yancheng Clinical College of Xuzhou Medical University, 166 West Yulong Road, Yancheng 224005, Jiangsu Province, and Department of Ultrasound, Yangpu Hospital, Tongji University School of Medicine, 450 Tengyue Road,

Shanghai 200090, People's Republic of China

E-mail: zxzshyzx@126.com

Phone/fax: 021-65690520-216

021-65690520-813 evaluation of cardiopulmonary function [1-8]. It is correlated with RV systolic and diastolic function, pulmonary vascular status (pulmonary vascular resistance) and left ventricular (LV) systolic and diastolic function. To date, PTT values have been measured by a variety of imaging methods, including contrast echocardiography, for most species, from cats and dogs to humans, with a high reliability, repeatability and accuracy [1-9]. But, the values of PTT in rats have not been reported.

It is well known that any cardiopulmonary medication may produce an effect on cardiopulmonary function and pulmonary vascular status, which may further result in the changes of PTT. Adenosine (Ade) and isoproterenol (Iso) are two different kinds of cardiopulmonary medication. Studies have shown that consecutive treatment of isolated heart with a high dose of Iso and Ade (Iso/Ade treatment) confers strong protection against ischaemia/reperfusion injury during heart surgery $[10,11]$. 
Regarding the kind of change of PTT they bring about in vivo remains unknown. In this paper the effects of Ade, Iso and their combinations on PTT in a rat model using contrast echocardiography were compared.

\section{Materials and methods}

\section{Contrast agent, adenosine and isoproterenol preparation}

A commercially available second-generation contrast agent, SonoVue ${ }^{\circledR}$ phospholipid-shell sulfur hexafluoride microbubbles (Bracco, Milan, Italy) was used for contrast echocardiography. A total of $59 \mathrm{mg}$ of SonoVue ${ }^{\circledR}$ was diluted in $5 \mathrm{~mL}$ of saline according to the manufacturer's protocol. Adenosine dry powder (Sigma, Shanghai, China) was diluted with normal saline to $0.46 \mathrm{uM} / \mathrm{mL}$, and isoproterenol solution (Harvest Pharma, Shanghai, China) was diluted with normal saline to $2.4 \mathrm{uM} / \mathrm{mL}$.

\section{Equipment}

The conventional and contrast echocardiographic data were acquired with a commercially available ultrasound system (Mindray M9, Mindray Medical Systems, Shenzhen, China) equipped with a L12-4s transducer (4-8 MHz), using a multi-pulse contrast-specific imaging protocol (power modulation imaging) combined with pulse inversion power Doppler.

\section{Animal preparation and grouping}

Thirty-two adult SD rats (male, 12 15 weeks old, weighing 250 400g; SLACCAS, Shanghai, China) were enrolled in the present study, with the approval of Animal Care and Use Committee, Xuzhou Medical University and Tongji University School of Medicine. These rats were divided into four groups $(\mathrm{n}=8)$ according to the medicines of tail-intravenous injection: Group 1 received $0.5 \mathrm{ml}$ of sterile pyrogen-free normal saline rapidly injected within $3 \mathrm{~s}$ at first and then $0.5 \mathrm{ml}$ microbubbles maintained $(0.25 \mathrm{~mL} / \mathrm{min})$ for 2 minutes (control group); Group 2 received $0.5 \mathrm{ml}$ adenosine solution $(0.23 \mathrm{uM})$ rapidly injected within $3 \mathrm{~s}$ at first and then $0.5 \mathrm{ml}$ microbubbles maintained $(0.25 \mathrm{~mL} / \mathrm{min})$ for 2 minutes (Aden group); Group 3 received $1 \mathrm{ml}$ isoproterenol solution (2.4 $\mathrm{uM}$ ) rapidly injected within $3 \mathrm{~s}$ at first and then $0.5 \mathrm{ml}$ microbubbles maintained $(0.25 \mathrm{~mL} / \mathrm{min})$ for 2 minutes (Iso group); and Group 4 received $0.5 \mathrm{ml}$ adenosine solution $(0.23 \mathrm{uM})+1 \mathrm{ml}$ isoproterenol solution $(2.4 \mathrm{uM})$ consecutively within $3 \mathrm{~s}$ at first and then $0.5 \mathrm{ml}$ microbubbles maintained $(0.25 \mathrm{~mL} / \mathrm{min})$ for 2 minutes (Aden+Iso group).

\section{D ultrasound examination}

SD rats were anesthetized by intraperitoneal injection of $10 \%$ chloral hydrate $(350 \mathrm{mg} / \mathrm{kg}$ body weight). The echocardiographic study was performed in the supine position. From parasternal short axis view, left ventricular maximal diameter at end-diastole and end-systole ( LVd and LVs), right ventricular diameter at end-diastole and end-systole (RVd and RVs), interventricular septum thickness at end-diastole and end-systole (IVSTd and IVSTs), right ventricular free wall thickness at enddiastole and end-systole (RVFWTd and RVFWTs) were measured. The interventricular septum systolic thickening rate (IVSSTR), right ventricular free wall systolic thickening rate (RVFWSTR) was calculated and left ventricular ejection fraction (LVEF) was obtained by real time $2 \mathrm{D}$ echo method.

\section{Real time contrast echocardiography}

All examinations were done by a single physician experienced with contrast echocardiography. Examination was performed from the parasternal short axis view. Gain, depth, transmit focus and post-processing were optimized at the beginning of the study and held constant throughout. The optimal balance between myocardial contrast enhancement and attenuation in our setting was achieved at a low mechanical index of 0.086 (acoustic power $7.8 \%$, thermal index of soft tissue 0.0 ). The whole process of ventricular enhancement, i.e., from the gradually visible opacification to the plateau, then to the attenuation, about 120 s, was stored digitally.

\section{PTT measurement}

Image analysis was performed off-line by two experienced operators (fig 1). The clip containing contrast agent entry and passage through the heart was carefully reviewed. Three kinds of PTT were calculated. Onset $\mathrm{RV}^{-}$ Onset $_{\text {LV }}$ PTT (PTT1) was calculated as the number of seconds from the first frame on which the contrast agent started to fill right ventricular to the first frame on which the contrast agent reached the left ventricular by dividing this frame count by the frame rate. Peak $_{\mathrm{RV}}-\mathrm{Peak}_{\mathrm{LV}}$ PTT (PTT2) was calculated as the number of seconds from the time of the peak opacification in the right ventricle to the time of the peak opacification in the left ventricle determined by the time-intensity curves of contrast echocardiography. Onset $\mathrm{RV}_{\mathrm{RV}}-$ peak $_{\mathrm{LV}}$ PTT (PTT3) was calculated as the number of seconds from the time of the initial appearance of contrast agent in the right ventricle to the peak opacification in the left ventricle determined by the time-intensity curves of contrast echocardiography.

Intraobserver and interobserver variability for identification of PTT1, PTT2 and PTT3 were obtained by repeated, blinded analysis of 10 randomly selected rats after a minimum time interval of 2 weeks.

\section{Statistical analysis}

Results are expressed as mean \pm standard deviation. The differences among groups were tested using oneway ANOVA. A receiver operating characteristic curve 

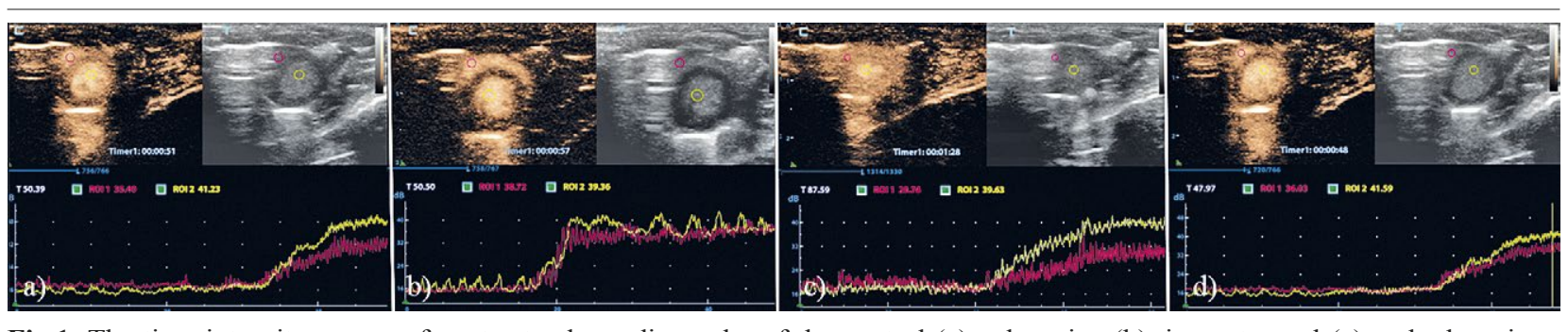

Fig 1. The time-intensity curves of contrast echocardiography of the control (a), adenosine (b), isoproterenol (c) and adenosine+ isoproterenol (d) group.

(ROC) analysis of was used to compare the performance of PTT based on different calculation methods in differentiating Aden or/and Iso group from control subjects, determine the optimal cut-off points and validity parameters. Bland \& Altman was used to measure the inter/ intra observer variability. A value of $p<0.05$ was considered statistically significant. All statistical analysis was performed with SPSS version 16 software for Windows (SPSS Inc, Chicago, IL).

\section{Results}

\section{Tolerability and contrast echocardiography quality}

All rats could tolerate the dosage of Ade or/and Iso injected through the tail vein. No adverse reactions were observed. Using the high frequency transducer and the dosage of Sonovue mentioned above, good image quality was achieved in all rats.

\section{Echocardiographic and hemodynamic \\ characteristics}

As shown in Table I, with Ade injection, heart rate increased significantly. Compared to the control group, the systolic cardiac chambers became smaller, the myo- cardium became thinner, systolic thickening rate became greater and LVEF increased significantly. With Iso injection, the heart rate increased more significantly (nearly $500 \mathrm{bpm}$ ). Compared to the control group, the systolic cardiac chambers became even smaller, the myocardium thickened at the end of systole and the LVEF increased more significantly. But, the RVd and the difference between RVd and RVs became smaller. In addition, systolic thickening rate in the Iso group was slightly lower than in the Ade group. With a combination injection of Ade and Iso, there was no change in heart rate. Compared to the control group, the cardiac size, myocardium thickness, systolic thickening rate, LVEF and RV function did not change significantly, too.

\section{PTT values}

As shown in figure 2, whether it was PTT1,PTT2 or PTT3, with the Ade injection, they all significantly declined (PTT1: from $0.99 \pm 0.39 \mathrm{~s}$ to $0.72 \pm 0.26 \mathrm{~s}, \mathrm{p}<0.05$; PTT2: from $4.77 \pm 2.39 \mathrm{~s}$ to $2.44 \pm 0.95 \mathrm{~s}, \mathrm{p}<0.01$; PTT3: from $32.71 \pm 9.31 \mathrm{~s}$ to $16.59 \pm 5.23 \mathrm{~s}, \mathrm{p}<0.001)$. With the Iso injection, however, they all significantly lengthened (PTT1: from $0.99 \pm 0.39 \mathrm{~s}$ to $2.87 \pm 0.59 \mathrm{~s}, \mathrm{p}<0.001$; PTT2: from $4.77 \pm 2.39 \mathrm{~s}$ to $9.23 \pm 5.71 \mathrm{~s}, \mathrm{p}<0.01$; РTT3:

Table I. Comparison of echocardiographic parameters in control, adenosine (Ade), isoproterenol (Iso) and adenosine and isoproterenol combined treatment (Ade+Iso) groups

\begin{tabular}{|c|c|c|c|c|}
\hline Variables & Control & Ade & Iso & Ade+Iso \\
\hline $\mathrm{HR}, \mathrm{bpm}$ & $402.20 \pm 9.26$ & $447.00 \pm 7.75^{* *}$ & $503.67 \pm 3.21 * * \#$ & $409.40 \pm 7.56$ \\
\hline $\mathrm{RVd}, \mathrm{mm}$ & $3.17 \pm 0.65$ & $3.01 \pm 0.22$ & $1.77 \pm 0.15 * * \#$ & $3.22 \pm 0.58$ \\
\hline $\mathrm{RVs}, \mathrm{mm}$ & $2.37 \pm 0.15$ & $1.70 \pm 0.36^{* *}$ & $1.53 \pm 0.06$ & $2.34 \pm 0.19$ \\
\hline RVFWTd, mm & $0.67 \pm 0.06$ & $0.30 \pm 0.10 * *$ & $0.53 \pm 0.06^{* \# \#}$ & $0.64 \pm 0.07$ \\
\hline RVFWTs, mm & $0.90 \pm 0.11$ & $0.53 \pm 0.06^{* *}$ & $0.97 \pm 0.06^{\# \#}$ & $0.87 \pm 0.13$ \\
\hline RVFWSTR, \% & $35.72 \pm 18.89$ & $91.67 \pm 62.91$ & $82.22 \pm 16.17 * * \#$ & $35.97 \pm 19.14$ \\
\hline $\mathrm{LVd}, \mathrm{mm}$ & $6.30 \pm 0.10$ & $6.10 \pm 0.09 *$ & $6.23 \pm 0.25$ & $6.33 \pm 0.14$ \\
\hline LVs, mm & $5.65 \pm 0.05$ & $4.07 \pm 0.40^{* *}$ & $3.30 \pm 0.02 * * \#$ & $5.59 \pm 0.07$ \\
\hline LVEF (\%) & $63.00 \pm 1.00$ & $80.01 \pm 4.24 * *$ & $92.50 \pm 2.12 * * \#$ & $64.35 \pm 1.98$ \\
\hline IVSTd, mm & $1.20 \pm 0.10$ & $0.73 \pm 0.15 * *$ & $1.13 \pm 0.12 * \#$ & $1.18 \pm 0.14$ \\
\hline IVSTs, mm & $1.73 \pm 0.06$ & $1.33 \pm 0.06^{* *}$ & $2.03 \pm 0.09 * \#$ & $1.76 \pm 0.09$ \\
\hline IVSSTR, \% & $45.36 \pm 16.74$ & $85.98 \pm 30.56^{* *}$ & $78.83 \pm 27.74^{* * \#}$ & $45.78 \pm 15.92$ \\
\hline
\end{tabular}

$* \mathrm{p}<0.05,{ }^{*} \mathrm{p}<0.01$ vs. control group; $\# \mathrm{p}<0.05, \# \# \mathrm{p}<0.01$ vs. Aden group. Data are expressed as mean \pm standard deviation. Bpm, beat per minute; $d$, at end-diastole; HR, heart rate; IVSSTR, interventricular septum systolic thickening rate; IVST, interventricular septum thickness; LV, left ventricular; RV, right ventricular; RVFWSTR, right ventricular free wall systolic thickening rate; RVFWT, right ventricular free wall thickness; s, at end-systole 
a)

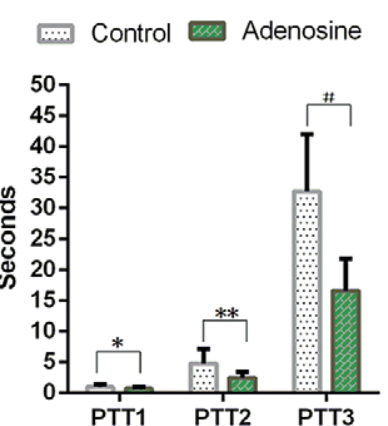

Control

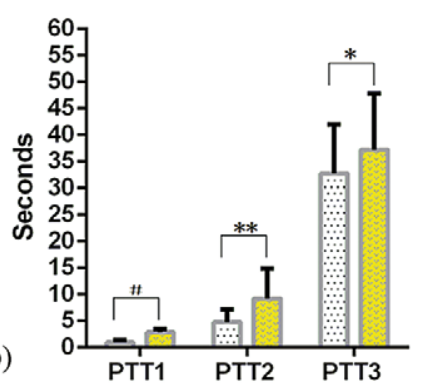

Control

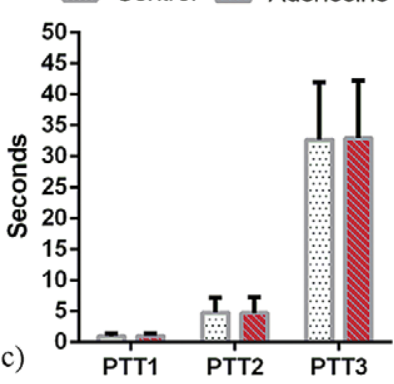

Fig 2. Comparison of pulmonary transit times among different groups. ${ }^{*} p<0.05$, ${ }^{*}{ }^{*} p<0.01$, \# $p<0.001$, vs. the control group. $\mathrm{PTT}_{1}$ Onset $_{\mathrm{RV}}-$ Onset $_{\mathrm{LV}}$ pulmonary transit time (the time required for a volume of contrast to travel from the initial appearance in the right ventricle to the initial appearance in the left atrium or left ventricle) determined by reviewing the contrast echocardiography in a "frame-by-frame" manner; PTT2, Peak $\mathrm{RV}^{-} \mathrm{Peak}_{\mathrm{LV}}$ pulmonary transit time (the time required for a volume of contrast to travel from the peak opacification in the right ventricle to the peak opacification in the left ventricle determined by the time-intensity curves of contrast echocardiography); PTT3, Onset ${ }_{\mathrm{RV}}-\mathrm{Peak}_{\mathrm{LV}}$ pulmonary transit time (the time required for a volume of contrast to travel from the initial appearance in the right ventricle to the peak opacification in the left ventricle) determined by the time-intensity curves of contrast echocardiography.

from $32.71 \pm 9.31 \mathrm{~s}$ to $37.20 \pm 10.57 \mathrm{~s}, \mathrm{p}<0.05)$. Interestingly, with a combination injection of Ade and Iso, all the PTT values were the same as the control group's (PTT1:1.02 \pm 0.43 s vs. $0.99 \pm 0.39$ s, $>0.05$; PTT2: $4.73 \pm 2.51$ s vs. $4.77 \pm 2.39 \mathrm{~s}, \mathrm{p}>0.05$; PTT3: $32.94 \pm 9.35 \mathrm{~s}$ vs. $32.71 \pm 9.31 \mathrm{~s}, \mathrm{p}>0.05)$.

\section{ROC curve analysis}

Comparison of the performance of PTT based on different calculation methods in differentiating the Ade or/ and Iso group from the control subjects, in other words, which one was more susceptible to Ade or/and Iso among PTT1, PTT2 and PTT3, was shown in Table II. In the Ade group, the AUC, sensitivity and Youden's index were maximal in PTT3, i.e, Ade is most likely to cause changes in PTT3. In the Iso group, the AUC, specificity and Youden's index were all were maximal in PTT1: therefore, Iso is the most likely to cause changes in PTT1. In the Ade+ Iso group, each AUC was less than 0.5, i.e, the combined administration of Ade and Iso could not cause changes in any PTT.

\section{Reproducibility}

Bland-Altman analysis showed the interobserver and intraobserver agreement for the measurement of PTT values were very good: intraclass correlation coefficients were all $>0.97$ for interobserver and intraobserver variability.

Table II. Comparison of the performance of pulmonary transit time based on different calculation methods in differentiating adenosine (Ade) or/and isoproterenol (Iso) from control subjects

\begin{tabular}{llllll}
\hline Medicines & AUC & Sensitivity (\%) & Specificity (\%) & Youden's index & Cutoff value \\
\hline Ade & & & & & \\
PTT1 & $0.750(0.490-1.010)$ & 71 & 75 & 0.46 & 0.835 \\
PTT2 & $0.768(0.510-1.026)$ & 71 & 75 & 0.46 & 3.19 \\
PTT3 & $0.921(0.820-1.022)^{*}$ & $100^{*}$ & $81.4^{*}$ & $0.81^{*}$ & 31.78 \\
Iso & & & & & \\
PTT1 & $0.958(0.858-1.059)$ & 83.3 & $100^{*}$ & $0.833^{*}$ & 1.44 \\
PTT2 & $0.792(0.527-1.056)$ & $100^{*}$ & 75 & 0.75 & 8.41 \\
PTT3 & $0.500(0.176-0.824)$ & 50 & 75 & 0.25 & 38.78 \\
Ade+Iso & & & & & \\
PTT1 & $0.422(0.103-0.741)$ & 66.7 & 35.3 & 0.02 & 1.23 \\
PTT2 & $0.438(0.127-0.748)$ & 66.7 & 37.5 & 0.04 & 4.97 \\
PTT3 & $0.375(0.06-0.690)$ & 66.7 & 25 & -0.07 & 12.28 \\
\hline
\end{tabular}

${ }^{*} \mathrm{p}<0.05$, vs. the same validity parameters in the same medicines group. AUC, areas under receiver operating characteristic curve; PTT1,OnsetRV-OnsetLV pulmonary transit time (the time required for a volume of contrast to travel from the initial appearance in the right ventricle to the initial appearance in the left atrium or left ventricle) determined by reviewing the contrast echocardiography in a"frame-byframe" manner; PTT2, PeakRV-PeakLV pulmonary transit time (the time required for a volume of contrast to travel from the peak opacification in the right ventricle to the peak opacification in the left ventricle determined by the time-intensity curves of contrast echocardiography); PTT3, OnsetRV-peakLV pulmonary transit time (the time required for a volume of contrast to travel from the initial appearance in the right ventricle to the peak opacification in the left ventricle) determined by the time-intensity curves of contrast echocardiography. 


\section{Discussion}

This is the first report on the effects of Ade, Iso and their combinations on PTT in rats using contrast echocardiography. The results presented here indicate that Ade or/and Iso exerted distinct effects on PTT: Ade shortened PTT, Iso lengthened PTT and the combinations of Ade and Iso had no significant effect on PTT. Moreover, Ade had a greater effect on PTT3 while Iso had a greater effect on PTT1. These findings suggest that we need to consider the effects of medicine (especially cardiopulmonary vasoactive drugs) on the PTT values during their usage. It also provides the basis for the translation into clinical practice of consecutive Iso/Ade treatment from the perspective of pulmonary circulation.

Although PTT is defined as the time required for a volume of contrast to travel from RV to LA or LV. The concept is still not very clear now, because it does not specify when (initial or peak opacification?) starts in order to time when the contrast agent reaches the heart cavity (RV, LA or LV). Most studies take the time required for a volume of contrast to travel from RV peak opacification to LA (or LV) peak opacification as PTT, i.e., Peak $\mathrm{RV}_{\mathrm{RV}}-\mathrm{Peak}_{\mathrm{LV}}$ PTT (PTT2) in our study. There are also some studies that use the time required for a volume of contrast to travel from RV initial appearance to LA (or LV) the initial appearance as PTT, i.e., Onset $_{\mathrm{RV}}$-Onset $_{\mathrm{LV}}$ PTT (PTT1) in our study. There is another calculation of PTT: the time required for a volume of contrast to travel from RV initial appearance to LA (or LV) peak opacification, i.e. Onset ${ }_{\mathrm{RV}}-$ Peak $_{\mathrm{LV}}$ PTT (PTT3) in our study. PTT1 is believed to mainly reflect the pulmonary vascular status, such as intrapulmonary vasodilatation, pulmonary arteriovenous fistula and abnormal angiogenesis, directly connected arteries-veins, and so on. Our previous study confirmed PTT1 is a reliable marker for the differential diagnosis of pulmonary nodules [9]. PTT3 is believed to a very important potential parameter for the calculation of pulmonary vascular volume although some studies have used PTT2 rather than PTT3 as a reference to calculate it $[7,12]$. Therefore, in this study, three kinds of PTT were calculated for comparison.

Ade and Iso, in addition to routine clinical use, also used to mimic the potent cardioprotection of temperature preconditioning by consecutive activation of protein kinases $A$ and protein kinases $C[10,11]$. Moreover, Iso and Ade, alone or simultaneously, protected isolated rat hearts but the consecutive treatment gave the highest protection [13]. Such a strategy seems to be useful in cardiac surgery involving ischaemic cardioplegic arrest and cardiopulmonary bypass. However, in vivo, it may also bring about impacts on cardiopulmonary function and pulmo- nary vascular status, and then result in the PTT changes. In this study, the rats were divided into 4 groups of control, Ade, Iso, and Aden+Iso just to explore their effect on PTT in detail. The results showed that a shortened PTT was found in the Ade group, a lengthened PTT in the Iso group and an unchanged PTT in the Ade+Iso group. Its underlying mechanisms are not fully known and the possible mechanisms involved require to be further discussed.

Firstly, Ade and Iso, alone or simultaneously, have different effects on cardiac function. Through 2D echo examination, it was found that the ability of Ade to enhance RV contraction (represented by RVFWSTR and the difference of RVd and RVs) was greater than that of Iso, with a normal LV diastolic volume (represented by $\mathrm{LVd}$ ) and RV diastolic volume (i.e., the volume of systemic circulation back to the heart; represented by RVd). Enhanced right ventricular systolic function, normal RV diastolic volume and normal LV diastolic volume (equivalent to normal left atrial pressure) would result in a shortened PTT. The ability of Iso to enhance LV contraction (represented by LVEF and the difference of LVd and LVs) was greater than that of Ade, with a normal LV diastolic volume (represented by LVd), but with a reduced RV contractility (represented by the difference of RVd and RVs), a decrease in RV diastolic volume (represented by RVd) and with a thicker myocardium and impaired systolic thickening rate. Reduced right ventricular systolic function, decreased RV diastolic volume (equivalent to RV preload) and normal LV diastolic volume would result in a lengthened PTT. Compared to the control group, the combination of Ade and Iso had no significant effect on cardiac size, cardiac function and so on. Therefore, no changes were seen in PPT.

Secondly, Ade and Iso, alone or simultaneously, have different effects on pulmonary vascular status. In our study, Ade and Iso were all administered intravenously through the tail vein. Ade acted as a potent selective pulmonary vasodilator by increasing intracellular cAMP via A2 receptor agonism [14] because of its rapid endothelial metabolism [15]. Pulmonary angiectasia would result in a decreased pulmonary vascular volume and a shortened PTT. Iso, a non-selective $\beta$-adrenergic agonist, acting on the $\beta 2$ receptor of the bronchial smooth muscle, caused a strong relaxation effect on the bronchial smooth muscle, and relived bronchial spasm. However, the bronchial smooth muscle relaxation made airway resistance reduced, the latter further made ventilation perfusion ratio abnormal and hypoxemia aggravated, and finally caused a hypoxic pulmonary vasoconstriction. In this setting, PTT was significantly prolonged. As for the effects of Ade+Iso on pulmonary vascular status, they might cancel each other out, so PTT did not change. 
In our previous experimental studies (unpublished data), the dose of Ade and Iso was explored, and the dose gradient (Ade: $0.10,0.23,0.46$ and $0.92 \mathrm{uM} / \mathrm{mL}$; Iso: 1.2 , 2.4, 4.8, $9.6 \mathrm{uM} / \mathrm{mL}$ ) had been done, and each dose of Ade and Iso had similar effects on PTT. In this study, the optimal concentration (Ade: $0.46 \mathrm{uM} / \mathrm{mL}$; Iso: $2.4 \mathrm{uM} /$ $\mathrm{mL}$ ) was adopted. Our previous studies had explored the modalities of administration of Ade and Iso, too. Ade administered via the jugular vein was complicated and had a low success rate. Although intraperitoneal injection of Iso had a high success rate; it couldn't be administered simultaneously with Ade. Therefore, simultaneous administration of Ade and Iso through tail vein was the best option. Due to the small size and fast circulation of rats, Ade may be metabolized less than we think.

Even so, our study had several limitations that should be considered. First of all, doses of Ade and Iso used in this study were selected according to our previous studies, without reference to those in vitro studies (sequentially with $5 \mathrm{nM}$ Iso and $30 \mu \mathrm{M}$ Ade) $[10,11]$. Further systematic exploration is required. Next, Ade and Iso in this study were all administered through tail-intravenous injection rather than subcutaneous injection, intraperitoneal injection, heart cavity injection, pulmonary vascular injection and bronchial injection, etc. The conclusions about the effects of Ade and Iso, alone or simultaneously on PTT may be one-sided. Finally, we still need more systematic researchs, including pathology at least, to support our idea.

\section{Conclusion}

In this study, the effects of Ade, Iso and their combinations on PTT in a rat model using contrast echocardiography was compared. The findings demonstrated that Ade or/and Iso exerted distinct effects on PTT: Ade shortened PTT, Iso lengthened PTT, and the combinations of Ade and Iso had no significant effect on PTT. Although it had some limitations, this study still reminds us of the need to consider the effects of medicine (especially cardiopulmonary vasoactive drugs) on the PTT values. At the same time, it provides the basis for the clinical transformation of consecutive Iso/Ade treatment from the perspective of pulmonary circulation.

Acknowledgements: The authors gratefully acknowledge the assistance of Xiancheng Xia from the Department of Ultrasound, The Yancheng Clinical College of Xuzhou Medical University, Yancheng, Jiangsu Province, P.R. China.

Conflict of interest: none

\section{References}

1. Brittain EL, Doss LN, Saliba L, Irani W, Byrd BF 3rd, Monahan K. Feasibility and diagnostic potential of pulmonary transit time measurement by contrast echocardiography: a pilot study. Echocardiography 2015;32:1564-1571.

2. Herold IHF, Saporito S, Bouwman RA, et al. Reliability, repeatability, and reproducibility of pulmonary transit time assessment by contrast enhanced echocardiography. Cardiovasc Ultrasound 2016;14:1.

3. Monahan K, Coffin S, Lawson M, Saliba L, Rutherford R, Brittain E. Pulmonary transit time from contrast echocardiography and cardiac magnetic resonance imaging: Comparison between modalities and the impact of region of interest characteristics. Echocardiography 2019;36:119-124.

4. Zhao H, Tsauo J, Zhang X, et al. Pulmonary transit time derived from pulmonary angiography for the diagnosis of hepatopulmonary syndrome. Liver Int 2018;38:1974-1981.

5. Crosara S, Ljungvall I, Margiocco ML, Häggström J, Tarducci A, Borgarelli M. Use of contrast echocardiography for quantitative and qualitative evaluation of myocardial perfusion and pulmonary transit time in healthy dogs. Am J Vet Res 2012;73:194-201.

6. Streitberger A, Hocke V, Modler P. Measurement of pulmonary transit time in healthy cats by use of ultrasound contrast media "Sonovue": Feasibility, reproducibility, and values in 42 cats. J Vet Cardiol 2013;15:181-187.

7. Ali LA, Aquaro GD, Peritore G, et al. Cardiac magnetic resonance evaluation of pulmonary transit time and blood volume in adult congenital heart disease. J Magn Reson Imaging 2019;50:779-786.

8. Colin GC, Pouleur AC, Gerber BL, et al. Pulmonary hypertension detection by computed tomography pulmonary transit time in heart failure with reduced ejection fraction. Eur Heart J Cardiovasc Imaging 2020;21:1291-1298.

9. Wang B, Sun F, Zheng XZ, Sun CY.Anovel application of pulmonary transit time to differentiate between benign and malignant pulmonary nodules using myocardial contrast echocardiography. Int J Cardiovasc Imaging 2021;37:1215-1223.

10. Lewis M, Szobi A, Balaska D, et al. Consecutive Isoproterenol and Adenosine Treatment Confers Marked Protection against Reperfusion Injury in Adult but Not in Immature Heart: A Role for Glycogen. Int J Mol Sci 2018;19:494.

11. Khaliulin I, Halestrap AP, Bryant SM, Dudley DJ, James AF, Suleiman MS. Clinically-relevant consecutive treatment with isoproterenol and adenosine protects the failing heart against ischaemia and reperfusion. J Transl Med 2014;12:139.

12. Galanti G, Jayaweera AR, Villanueva FS, Glasheen WP, Ismail S, Kaul S. Transpulmonary transit of microbubbles during contrast echocardiography: implications for estimating cardiac output and pulmonary blood volume. J Am Soc Echocardiogr 1993;6:272-278.

13. Khaliulin I, Parker JE, Halestrap AP. Consecutive pharmacological activation of PKA and PKC mimics the potent cardioprotection of temperature preconditioning. Cardiovasc Res 2010;88:324-333. 
14. Aranda M, Bradford KK, Pearl RG. Combined therapy with inhaled nitric oxide and intravenous vasodilators during acute and chronic experimental pulmonary hypertension. Anesth Analg 1999;89:152-158.
15. Morgan JM, McCormack DG, Griffiths MJ, Morgan CJ, Barnes PJ, Evans TW. Adenosine as a vasodilator in primary pulmonary hypertension. Circulation 1991;84:11451149. 